\title{
Angiographic Morphology of Anterior Communicating Artery Aneurysms and Their Association with Rupture Risk
}

\author{
Veysel KAYA ${ }^{1}$, Mehmet KOLU ${ }^{2}$, Ahmet Turan KAYA ${ }^{3}$, Mehmet GEZER ${ }^{4}$, Kaya SARAC ${ }^{2}$, Bora TETiK ${ }^{5}$ \\ Ismail Okan YILDIRIM², Mehmet Akif DURAK ${ }^{5}$ \\ ${ }^{1}$ Kelkit State Hospital, Department of Radiology, Gumushane, Turkey \\ 2Inonu University Hospital, Department of Radiology, Malatya, Turkey \\ ${ }^{3}$ Amasya University, Department of Radiology, Amasya, Turkey \\ ${ }^{4}$ University of Health Sciences, Mehmet Akif Inan Training Research Hospital, Department of Radiology, Sanliurfa, Turkey \\ ${ }^{5}$ Inonu University Hospital, Department of Neurosurgery, Malatya, Turkey
}

Corresponding author: Veysel KAYA drvkvkfk@gmail.com

\section{ABSTRACT}

AIM: To estimate the rupture risk of anterior communicating artery (AComA) and AComA-related aneurysms according to their localization, angiographic architecture, and morphological features.

MATERIAL and METHODS: In this study, 124 patients with AComA and AComA-related anterior system aneurysms were retrospectively evaluated. The aneurysms were classified according to their morphological appearance and angiographic architecture. The size, size ratio, angiographic architecture, and aneurysmal dome orientation of ruptured and non-ruptured aneurysms were compared using digital subtraction angiography (DSA) 3D images.

RESULTS: There was a significant relationship between rupture risk and the size ratio $(p=0.043)$, morphological properties of the aneurysm $(p<0.001)$, aneurysm dome orientation (OR 1.29, 95\% Cl 1.32-6.818), and aneurysm type according to the angiographical architecture $(p<0.005)$.

CONCLUSION: In determining the rupture risk of AComA and AComA-related aneurysms, size alone is not a sufficient parameter with aneurysm morphology proving to be more efficacious. Grouping of aneurysms according to angioarchitecture, and its significant correlation with aneurysm rupture, may help to understand the underlying mechanisms in the formation and rupture of aneurysms. From this, more specific treatment protocols can be created, helping to improve the clinical evaluation of AComA aneurysms.

KEYWORDS: Aneurysm, Anterior communicating artery, Angiographical architecture, Morphology, Dome orientation

\section{INTRODUCTION}

Trefris ntracranial aneurysms occur in about $2 \%-5 \%$ of the Western population, of which approximately $30 \%$ Lof patients have multiple aneurysms (18). Anterior communicating artery (AComA) aneurysms constitute 30$37 \%$ of all intracranial aneurysms and are considered to be the most complex aneurysms. The incidence of subarachnoid hemorrhage in patients with an aneurysm is approximately $1-2 \%$ per year (10).
A greater number of unruptured aneurysms are now being diagnosed due to technological advances in imaging techniques (25). Although aneurysms are common, the rupture rate is low. However, morbidity and mortality rates are high in cases where the aneurysm does rupture. Therefore, it is critical that the risk factors of aneurysm rupture are identified.

The treatment of non-ruptured intracranial aneurysms remains controversial. The localization, size, and morphological features of aneurysms are thought to be risk factors of a rupture.
Veysel KAYA (1) : 0000-0002-7131-2277

Mehmet KOLU (D): 0000-0001-9659-6053

Ahmet Turan KAYA (1) : 0000-0001-9803-453X
Mehmet GEZER (10) : 0000-0003-4286-9583

Kaya SARAC (10): 0000-0001-5559-3219

Bora TETIK (D) : 0000-0001-7696-7785
Ismail Okan YILDIRIM (10) : 0000-0002-3641-0103 Mehmet Akif DURAK (1) : 0000-0003-0827-2708 
The international non-ruptured intracranial aneurysms working group (ISUIA) has provided guidelines based on the size and localization of unruptured aneurysms. Specific localizations have been found to be associated with aneurysm rupture (23). There have been contradictory findings in studies that have investigated the relationship between morphological and geometric characteristics and aneurysm rupture.

In this study, we aimed to identify the relationship between the aneurysm type and morphological features according to the angiographical architecture with AComA aneurysm rupture.

\section{MATERIAL and METHODS}

\section{Study Design and Patients}

The study was approved by the Inonu University Ethical Committee (2017/26-1). A retrospective analysis was performed using clinical and 3D digital subtraction angiography (DSA) data. The study included 124 patients (66 females and 58 males) who were diagnosed with AComA and AComArelated aneurysms between June 2014 and December 2018. A total of 86 patients with ruptured and 38 patients with unruptured AComA and AComA-related aneurysms were included in this study. Twenty percent of these patients had multiple aneurysms. Patients suspected of having mycotic, dissecting, and giant aneurysms were excluded, along with those patients under 18-years-old.

\section{Imaging}

DSA was performed using an AlluraXper FD20 monoplane unit (Philips Medical Systems, Best, The Netherlands). Rotational angiograms were obtained using a constant injection of contrast agent and a $240^{\circ}\left(+120^{\circ}-120^{\circ}\right)$ rotation with imaging at 116 frames for 5.1 seconds. Data from these images were transferred to the Integris Workstation (Philips Medical Systems, Best, The Netherlands) and reconstructed into 3D voxel data using the standard proprietary software provided. 3D digital reconstructions that did not contain the entire aneurysm or associated parent arteries were excluded. Patients DSA and 3D images were evaluated independently by two radiologists.

\section{Geometrical and Morphological Parameters}

a) Aneurysm size: Defined as the largest aneurysm diameter.

b) Height of aneurysm $(\mathbf{H})$ : The vertical distance between the aneurysm neck and the aneurysm dome was defined as the height of the aneurysm.

c) Aneurysm neck diameter: Defined as the neck width.

d) Maximal height (Hmax): Defined as the longest distance between the center of the aneurysm neck and the dome of the aneurysm.

e) Mean parent artery diameter: (Both $A_{1}$ diameter + Both $\mathbf{A}_{2}$ diameter) /4

f) Aspect ratio: H/aneurysm neck diameter

g) Size ratio: Hmax/average parent artery diameter

i) Direction of the aneurysm dome: anterior, posterior, superior, and inferior

\section{j) Inflow angle}

\section{k) Morphological characteristics (Figure 1)}

l) Angioarchitecture of aneurysm: We describe 10 different types of AComA and AComA-related aneurysms in all possible locations (Figure 2).

\section{m) Presence of ACA A1 segment aplasia-hypoplasia}

The statistical analysis was performed using Statistical Package for Social Sciences (SPSS) version 22.0 (SPSS, Chicago, IL, USA). Quantitative data was evaluated using the Kolmogorov Smirnov test. Normally distributed quantitative data were analyzed using a t-test and one-way analysis of variance (ANOVA). Data that were not normally distributed were analyzed using the Mann-Whitney $U$ and Kruskal Wallis tests. Qualitative data were assessed using the Chi-square test. A p-value of $<0.05$ was considered statistically significant.

\section{RESULTS}

The 3-D images of all patients were evaluated, and patients were divided into three groups according to the morphological features of the aneurysms. The rupture rate in patients with

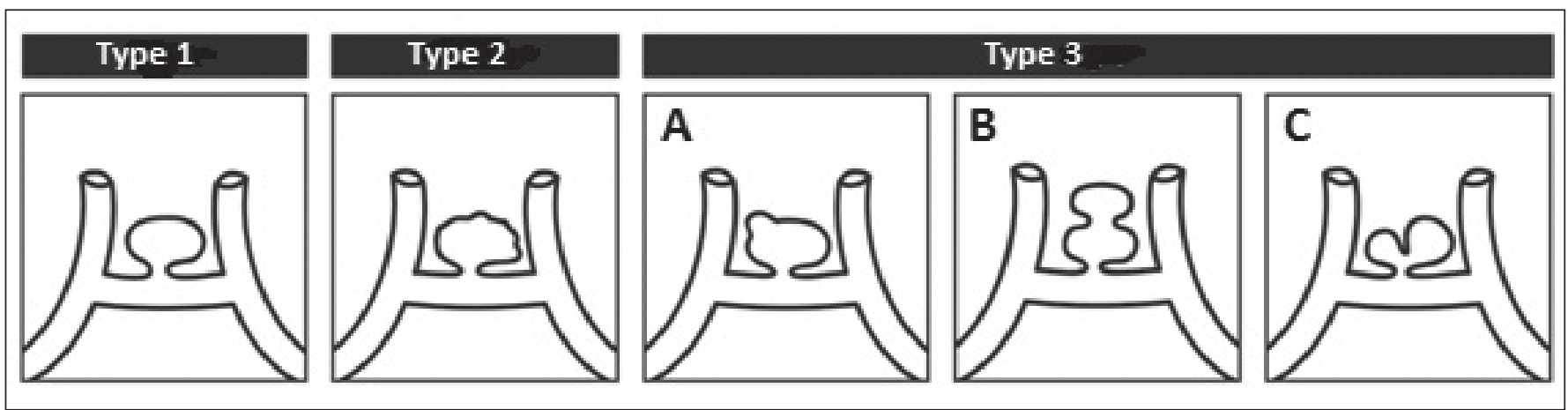

Figure 1: Classification based on the morphological features of Type 1. Well-circumscribed single saccular aneurysm, Type 2. Irregular bordered single saccular aneurysm, Type 3. Aneurysm with bleb formation (3A) and bilobed (3B) or multilobed (3C) aneurysm stemming from either the neck or body, which involves $25 \%$ of the total aneurysm. 

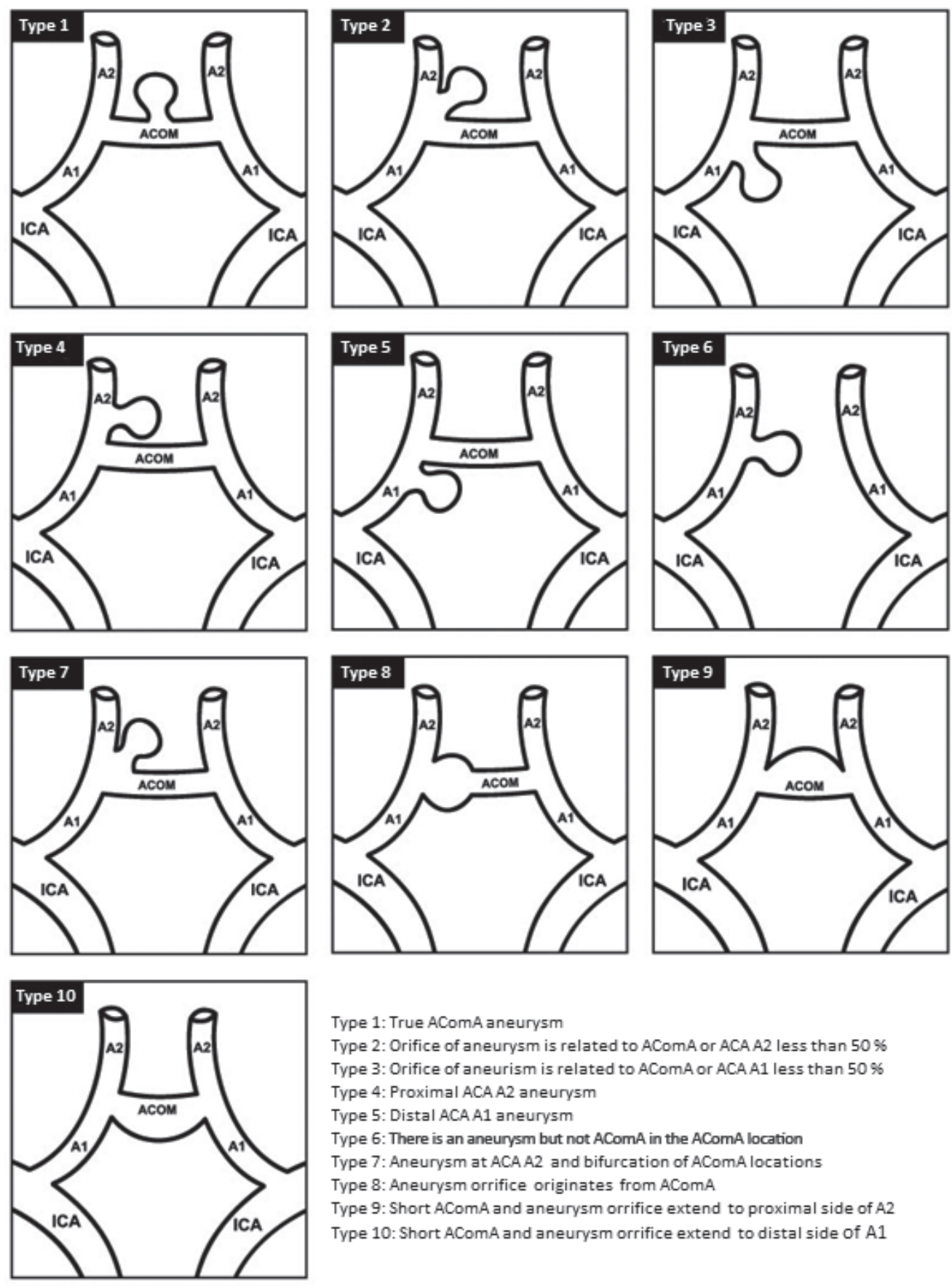

Type 1: True AComA aneurysm

Type 2: Orifice of aneurysm is related to AComA or ACA A2 less than $50 \%$ Type 3: Orifice of aneurism is related to AComA or ACAA1 less than $50 \%$ Type 4: Proximal ACA A2 aneurysm

Type 5: Distal ACAA1 aneurysm

Type 6: There is an aneurysm but not AComA in the AComA location

Type 7: Aneurysm at ACA A2 and bifurcation of AComA locations

Type 8: Aneurysm orrifice originates from AComA

Type 9: Short AComA and aneurysm orrifice extend to proximal side of $A 2$

Type 10: Short AComA and aneurysm orrifice extend to distal side of A1

Figure 2: Classification of anterior communicating artery (AComA) and AComA-related anterior system aneurysms according to their angiographic architecture.

type 2 and type 3 aneurysms was significantly higher than those with type 1 aneurysms $(p<0.001)$ (Figure 3$)$. Type 1 aneurysms were mostly less than $4 \mathrm{~mm}$, while $66.7 \%$ of type 2 aneurysms and $80 \%$ of type 3 aneurysms were larger than $4 \mathrm{~mm}(\mathrm{p}<0.001)$. In addition, type 2 and 3 aneurysms had a wider neck diameter $(p<0.001)$ (Figure 4). However, there was no significant correlation between the diameter of the mean parent artery and the morphological features $(p=0.646)$.
The vast majority of AComA aneurysms occurred in the anterior projection, with the lowest occurring in the inferior projection. AComA aneurysms with superior projections were found to have a higher rupture risk than those with anterior projections (OR 1.29, 95\% Cl 1.32-6.81) (Table I).

The patients were categorized into three groups $(<4 \mathrm{~mm}$, 4-7 $\mathrm{mm},>7 \mathrm{~mm}$ ) according to the aneurysm size. There 

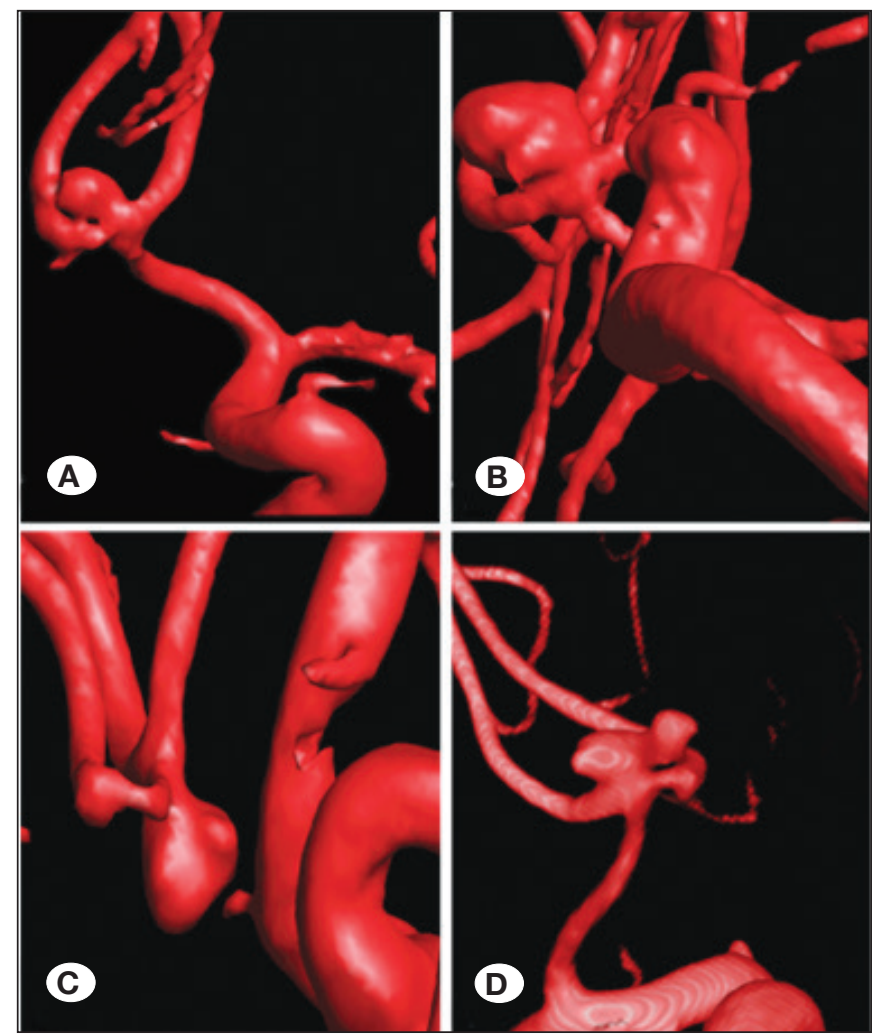

Figure 3: 3D images of patients aneurysms. A) Type 1. Wellcircumscribed single saccular aneurysm. B) Type 2. Irregular bordered single saccular aneurysm. C-D) Type 3-4. Aneurysm with bleb formation and bilobed or multilobed aneurysm.

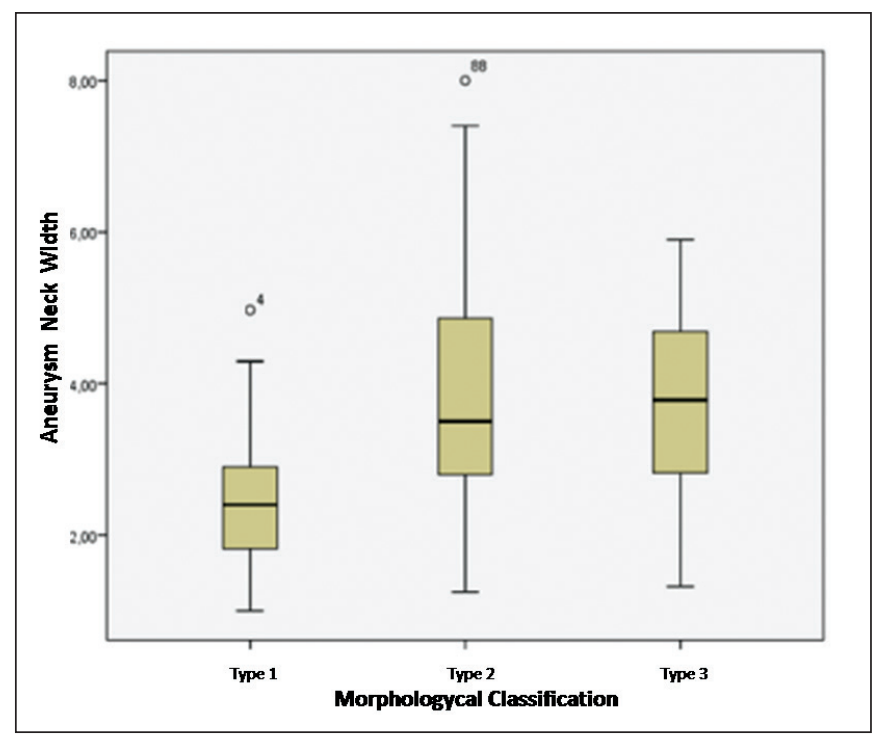

Figure 4: Correlation between the aneurysm neck width and types that were classified according to the morphological features of the aneurysm. was no significant relationship between the aneurysm size and ruptures $(p=0.962)$ (Table $\mathrm{I})$. In addition, there was no significant correlation between aneurysmal rupture and the aspect ratio, inflow angle, neck diameter, mean parent artery, and presence of ACA A1 hypoplasia-aplasia (Table I). However, the size ratio was higher in patients with ruptured aneurysms $(p=0.043)$. Furthermore, $29.8 \%$ and $31.6 \%$ of patients with AComA aneurysms had A1 segment aplasia and hypoplasia, respectively (Table I). In addition, 16 concomitant venous anomalies and 5 arterial structural abnormalities were observed. There was no significant correlation between the ruptured and unruptured groups $(p=0.123, p=0.113)$ (Table I).

We analyzed the DSA and $3 D$ images of the patients and classified the AComA and AComA-related anterior system aneurysms according to their angioarchitecture. The most frequently detected types were type 1,2, and 7 . Type 10 was not detected in any of the included patients (Table II). The majority of AComA and AComA-related anterior system aneurysms were bifurcation and bifurcation-related aneurysms.

In terms of rupture, there was a statistically significant difference between the types according to the aneurysm angioarchitecture $(p=0.005)$. The rupture rates were relatively higher than the unruptured rate in types 1, 4, 5, 6, and 7 (Table II). The unruptured rate was relatively higher than the ruptured rate in type 3 (Table II). These data show that the probability of a rupture is higher in types $1,4,5,6$, and 7 .

\section{DISCUSSION}

In this study, 10 aneurysm-related factors (size, neck width, aspect ratio, mean parent artery diameter, size ratio, inflow angle, angiographical architecture, morphologic properties, aneurysm dome orientation, and concomitant ACA A1 segment aplasia or hypoplasia) were investigated in ruptured and non-ruptured aneurysms. The relationship between these factors and rupture was retrospectively analyzed.

The relationship between ruptured intracranial aneurysms and aneurysm irregularities has been investigated in many recent studies. Bleb formation is the most frequently used parameter for aneurysm irregularity. In many clinical studies, intracranial aneurysms with one or more bleb formation have been reported to have a high rupture risk $(4,13,14)$. In this study, AComA and AComA-related aneurysms were examined in three separate groups according to their morphological features. There was a significant relationship between type 2 and 3 groups in terms of aneurysm irregularity and rupture. In the present study, a positive correlation was found between the aneurysm dimensions and aneurysm irregularity. At the same time, a statistically significant relationship between morphological typing and the aneurysm neck width was observed, and aneurysm irregularity increased as the neck width increased. This suggests that the aneurysm size and neck width may affect aneurysm irregularity, resulting in a rupture of the aneurysm.

Even though aneurysm size is shown to be one of the most important factors related to aneurysm rupture, the critical threshold size for aneurysm rupture is contentious 
$(10,11,16,19,22,26,29)$. Aneurysm localization is an independent factor of rupture risk and may be an important factor to consider in discussions on size. It is accepted that the aneurysms localized at AComA and posterior communicating artery (PComA) are more likely to rupture than aneurysms localized in other parts of the anterior circulation system
$(2,10,12)$. Some studies have reported that the relationship between aneurysm size and bleeding risk is closely related to localization (9). In this study, AComA and AComA-related aneurysms were examined in three size groups: $<4 \mathrm{~mm}, 4-7$ $\mathrm{mm}$, and $>7 \mathrm{~mm}$. No correlation was found between the groups in terms of rupture.

Table I: Geometrical and Morphological Parameters of the Patients

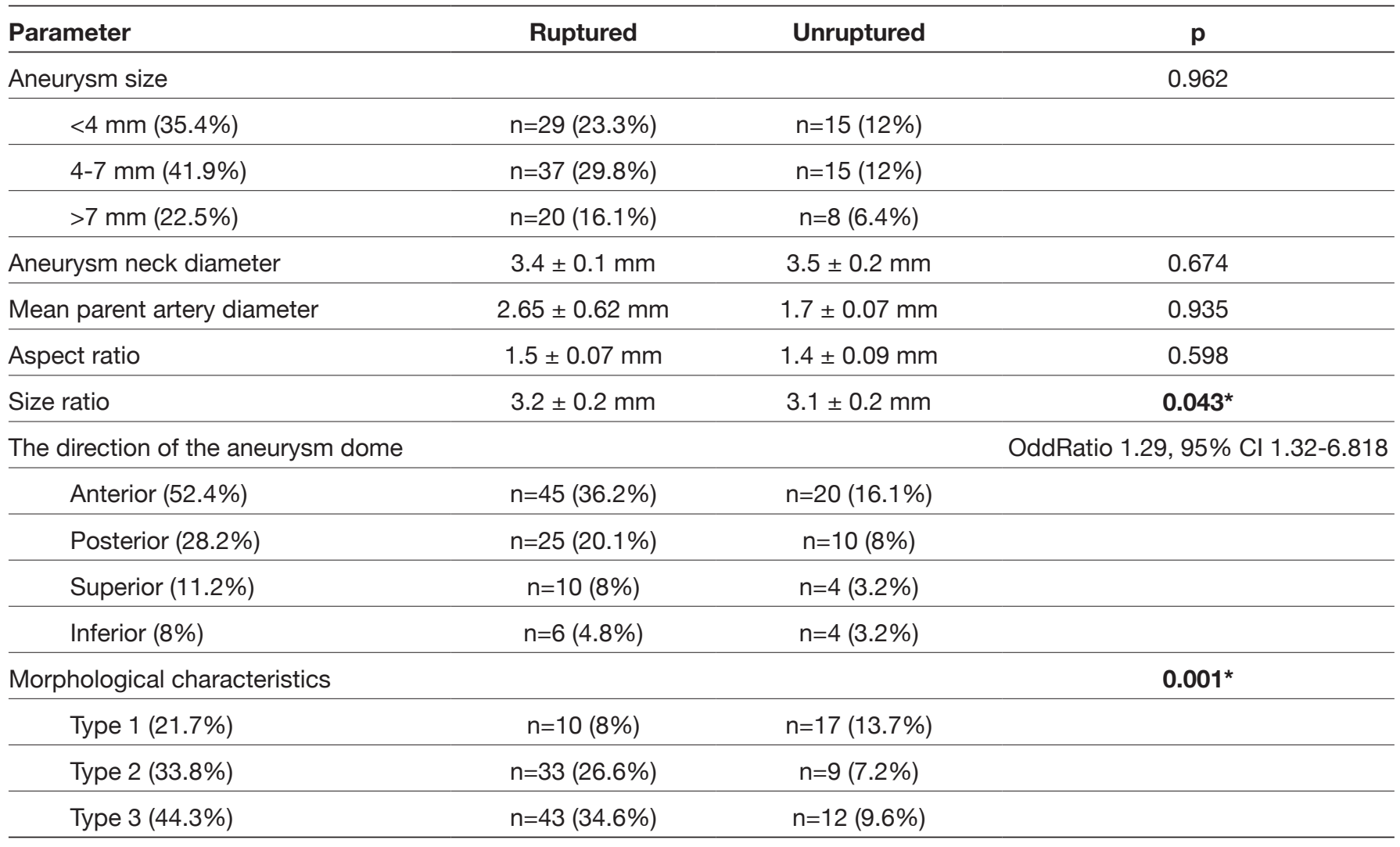

( $p<0.05$, statistically significant).

Table II: The Distribution of Aneurysm Rates in Different Types According to the Aneurysm Architecture

\begin{tabular}{|c|c|c|c|c|}
\hline & & Rupture (-) & Rupture (+) & Total \\
\hline \multirow{10}{*}{$\begin{array}{l}\text { Architecture of the } \\
\text { aneurysm }\end{array}$} & Type 1 & $3(2.4 \%)$ & 19 (15.3\%) & $22(17.7 \%)$ \\
\hline & Type 2 & $8(6.4 \%)$ & 24 (19.3\%) & $32(25.8 \%)$ \\
\hline & Type 3 & $9(7.2 \%)$ & $2(1.6 \%)$ & $11(8.8 \%)$ \\
\hline & Type 4 & $1(0.8 \%)$ & $6(4.8 \%)$ & $7(5.6 \%)$ \\
\hline & Type 5 & $2(1.6 \%)$ & $5(4 \%)$ & $7(5.6 \%)$ \\
\hline & Type 6 & $1(0.8 \%)$ & $3(2.4 \%)$ & $4(3.2 \%)$ \\
\hline & Type 7 & 13 (10.4\%) & $26(20.9 \%)$ & 39 (31.4\%) \\
\hline & Type 8 & 0 & $1(0.8 \%)$ & $1(0.8 \%)$ \\
\hline & Type 9 & $1(0.8 \%)$ & 0 & $1(0.8 \%)$ \\
\hline & Type 10 & 0 & 0 & 0 \\
\hline Total & & 38 (30.6\%) & $86(69.3 \%)$ & $124(100 \%)$ \\
\hline
\end{tabular}


Some studies have reported that the hemodynamics of intracranial aneurysms strongly depends on the geometry of the aneurysm sac and feeding vessels $(6,7)$. Several geometric parameters such as aspect ratio, size ratio, inflow angle, and aneurysm dome orientation have been shown to be more related to aneurysm rupture than aneurysm size. However, there are no effective methods for predicting aneurysm rupture in asymptomatic patients $(5,10,17,28)$. Besides, some studies reported an increased risk of rupture due to increased flow stress on the dominant side in cases where there is a $50 \%$ or greater difference in diameter between the two $\mathrm{A} 1$ segments, A1 segment aplasia or hypoplasia $(6,8,24,27)$.

AComA and AComA-related aneurysms have a more complex structure than other anterior system aneurysms due to vascular anatomy, hemodynamics of the AComA, the relative dominance of the A1 segments, and different configurations of the $\mathrm{A} 2$ segments. Due to this, AComA aneurysms may originate from different localizations of this complex artery $(3,15)$. To our knowledge, no study has described the angioarchitecture of AComA aneurysms and examined the relationship to rupture risk. Some studies on PComA aneurysms have reported differences in angioarchitecture between ruptured and nonruptured aneurysms $(1,20,21)$. To examine the frequency of different AComA aneurysm configurations and whether they affect aneurysm bleeding, we analyzed 3D and DSA images. Then, we divided AComA and AComA-related aneurysms into 10 different types according to the angioarchitecture. We found a statistically significant difference between the groups in relation to the occurrence of a rupture $(p=0.005)$. In particular, types $1,4,5,6$, and 7 , had a higher risk of rupture. Contrastingly, type 3 had a low risk of rupture. Our results can significantly contribute to determining the rupture risk of AComA aneurysms and should be supported by further studies with a larger sample size.

There are a few limitations to this study. This single-center retrospective study was conducted on a relatively small group of patients. In the cluster we examined, the number of ruptured aneurysms was slightly more than twice the number of non-ruptured aneurysms which may cause a selection bias. In addition, ruptured aneurysms may be affected by changes in shape that can occur during vasospasm or bleeding.

\section{CONCLUSION}

In determining the rupture risk of AComA and AComA-related aneurysms, size alone is not a sufficient parameter, with aneurysm irregularity being more efficacious.

Grouping of aneurysms according to angioarchitecture, and its significant correlation with aneurysm rupture, may help to understand the underlying mechanisms in the formation and rupture of aneurysms. This should help to create more specific treatment protocols, helping to improve their clinical evaluation.

\section{- REFERENCES}

1. Ambekar S, Madhugiri V, Bollam P, Nanda A: Morphological differences between ruptured and unruptured basilar bifurcation aneurysms. J Neurol Surg B Skull Base 74(2):9196, 2013

2. Baumann F, Khan N, Yonekawa Y: Patient and aneurysm characteristics in multiple intracranial aneurysms. Acta Neurochir Suppl 103:19-28, 2008

3. Castro MA, Putman CM, Sheridan MJ, Cebral CR: Hemodynamic patterns of anterior communicating artery aneurysms: A possible association with rupture. AJNR Am J Neuroradiol 30(2):297-302, 2009

4. Cebral JR, Sheridan M, Putman CM: Hemodynamics and bleb formation in intracranial aneurysms. AJNR Am J Neuroradiol 31(2):304-310, 2010

5. Dhar S, Tremmel M, Mocco J, Kim M, Yamamoto J, Siddiqui $\mathrm{AH}$, Hopkins LN, Meng $\mathrm{H}$ : Morphology parameters for intracranial aneurysm rupture risk assessment. Neurosurgery 63(2):185-196; discussion 196-197, 2008

6. Hassan T, Timofeev EV, Saito T, Shimizu H, Ezura M, Matsumoto Y, Takayama K, Tominaga T, Takahashi A: A proposed parent vessel geometry-based categorization of saccular intracranial aneurysms: Computational flow dynamics analysis of the risk factors for lesion rupture. J Neurosurg 103(4):662-680, 2005

7. Hoi Y, Meng H, Woodward SH, Bendok BR, Hanel RA, Guterman LR, Hopkins LN: Effects of arterial geometry on aneurysm growth: Three-dimensional computational fluid dynamics study. J Neurosurg 101(4): 676-681, 2004

8. Inagawa T: Risk factors for the formation and rupture of intracranial saccular aneurysms in Shimane, Japan. World Neurosurg 73(3):155-164; discussion e23, 2010

9. Juvela S, Porras M, Poussa K: Natural history of unruptured intracranial aneurysms: Probability of and risk factors for aneurysm rupture. J Neurosurg 93(3):379-387, 2000

10. Juvela S, Poussa K, Lehto H, Porras M: Natural history of unruptured intracranial aneurysms: A long-term follow-up study. Stroke 44(9):2414-2421, 2013

11. Kassell NF, Torner JC, Haley EC Jr, Jane JA, Adams HP, Kongable GL: The international cooperative study on the timing of aneurysm surgery. Part 1: Overall management results. J Neurosurg 73(1):18-36, 1990

12. Li M, Jiang Z, Yu H, Hong T: Size ratio: A morphological factor predictive of the rupture of cerebral aneurysm? Can J Neurol Sci 40(3):366-371, 2013

13. Mackey J, Brown Jr RD, Moomaw CJ, Sauerbeck L, Hornung R, Gandhi D, Woo D, Kleindorfer D, Flaherty ML, Meissner I, Anderson C, Connolly ES, Rouleau G, Kallmes DF, Torner J, Huston 3rd J, Broderick JP, FIA and ISUIA Investigators: Unruptured intracranial aneurysms in the Familial Intracranial Aneurysm and International Study of Unruptured Intracranial Aneurysms cohorts: Differences in multiplicity and location. J Neurosurg 117(1): 60-64, 2012

14. Matsukawa H, Fujii M, Akaike G, Uemura A, Takahashi O, Niimi Y, Shinoda M: Morphological and clinical risk factors for posterior communicating artery aneurysm rupture. J Neurosurg 120:104-110, 2014 
15. Matsukawa H, Uemura A, Fujii M, Kamo M, Takahashi $O$, Sumiyoshi S: Morphological and clinical risk factors for the rupture of anterior communicating artery aneurysms. J Neurosurg 118(5):978-983, 2013

16. Nader-Sepahi A, Casimiro M, Sen J, Kitchen ND: Is aspect ratio a reliable predictor of intracranial aneurysm rupture? Neurosurgery 54(6):1343-1347; discussion 1347-1348, 2004

17. Rahman M, Smietana J, Hauck E, Hoh B, Hopkins N, Siddiqui A, Levy El, Meng H, Mocco J: Size ratio correlates with intracranial aneurysm rupture status: A prospective study. Stroke 41(5):916-920, 2010

18. Rinkel GJ, Djibuti M, Algra A, van Gijn J: Prevalence and risk of rupture of intracranial aneurysms: A systematic review. Stroke 29:251-256, 1998

19. Rinkel GJE: Natural history, epidemiology and screening of unruptured intracranial aneurysms. J Neuroradiol 35(2):99103, 2008

20. Sadatomo T, Yuki K, Migita K, Taniguchi E, Kodama Y, Kurisu K: Morphological differences between ruptured and unruptured cases in middle cerebral artery aneurysms. Neurosurgery 62(3):602-609, 2008

21. Sadatomo T, Yuki K, Migita K, Taniguchi E, Kodama Y, Kurisu $\mathrm{K}$ : The characteristics of the anterior communicating artery aneurysm complex by three-dimensional digital subtraction angiography. Neurosurg Rev 29(3):201-207, 2006

22. Schievink WI, Piepgras DG, Wirth FP: Rupture of previously documented small asymptomatic saccular intracranial aneurysms. Report of three cases. J Neurosurg 76(6):10191024, 1992

23. UCAS Japan Investigators; Morita A, Kirino T, Hashi K, Aoki N, Fukuhara S, Hashimoto N, Nakayama T, Sakai M, Teramoto A, Tominari S, Yoshimoto T: The natural course of unruptured cerebral aneurysms in a Japanese cohort. $\mathrm{N}$ Engl $\mathrm{J}$ Med 366(26):2474-2482, 2012
24. Velthuis BK, van Leeuwen MS, Witkamp TD, Ramos LM, Berkelbach van der Sprenkel JW, Rinkel GJ: Surgical anatomy of the cerebral arteries in patients with subarachnoid hemorrhage: Comparison of computerized tomography angiography and digital subtraction angiography. J Neurosurg 95(2):206-212, 2001

25. Vlak MHm, Algra A, Brandenburg R, Rinkel GJ: Prevalence of unruptured intracranial aneurysms, with emphasis on sex, age, comorbidity, country, and time period: A systematic review and meta-analysis. Lancet Neurol 10(7):626-636, 2011

26. Wiebers DO, Whisnant JP, Huston J 3rd, Meissner I, Brown RD, Jr, Piepgras DG, Forbes GS, Thielen K, Nichols D, O'Fallon WM, Peacock J, Jaeger L, Kassell NF, Kongable-Beckman GL, Torner JC, International Study of Unruptured Intracranial Aneurysms Investigators. Unruptured intracranial aneurysms: Natural history, clinical outcome, and risks of surgical and endovascular treatment. Lancet 362(9378):103-110, 2003

27. Yasargil MG, Fox JL, Ray MW: The operative approach to aneurysms of the anterior communicating artery. Advances and Technical Standards in Neurosurgery 2:113-170, 1975

28. Yasuda R, Strother CM, Taki W, Shinki K, Royalty K, Pulfer K, Karmonik C: Aneurysm volume-to-ostium area ratio: $\mathrm{A}$ parameter useful for discriminating the rupture status of intracranial aneurysms. Neurosurgery 68:310-317; discussion 317-318, 2011

29. Yasui N, Magarisawa S, Suzuki A, Nishimura H, Okudera T, Abe T. Subarachnoid hemorrhage caused by previously diagnosed, previously unruptured intracranial aneurysms: A retrospective analysis of 25 cases. Neurosurgery 39:10961100; discussion 1100-1101, 1996 